\title{
Ending AIDS: All Hands on Deck
}

\author{
Ron Stall $^{1,2} \cdot$ James E. Egan ${ }^{1,2} \cdot$ Michael Cowing ${ }^{3}$
}

Published online: 22 October 2016

(c) Springer Science+Business Media New York 2016

We cannot end AIDS without building the capacity of gay men, other men who have sex with men and transgender populations in low-and middleincome countries to effectively fight the HIV epidemic within their own

communities.

This special issue of AIDS and Behavior presents a series of scientific papers written by a remarkable group of early career investigators from low- and middle-income countries (LMIC) across the globe. More impressive still, these investigators-which include gay men, other men who have sex with men, and transgender individuals (GMT) often come from settings that routinely discount their very existence and value as people, and in which repression, discrimination and violence are the bywords of their daily lives. Their ability to conduct community-focused HIV research within such settings, and then produce and publish papers in a respected scientific journal is a profound testament to the courage, commitment and hard work that each of these investigators brought to the innovative initiative- the HIV Scholars Program-profiled in this publication. This program is the result of an ambitious partnership between the Center for LGBT Health Research at the University of Pittsburgh Graduate School of Public Health, and amfAR, The Foundation for AIDS Research,

Ron Stall

rstall@pitt.edu

1 Department of Behavioral and Community Health Sciences, Graduate School of Public Health, University of Pittsburgh, A211 Crabtree Hall, 130 DeSoto Street, Pittsburgh, PA 15261, USA

2 Center for LGBT Health Research, Graduate School of Public Health, University of Pittsburgh, A211 Crabtree Hall, 130 DeSoto Street, Pittsburgh, PA 15261, USA

3 amfAR, The Foundation for AIDS Research, New York, NY, USA but it is the scholars themselves who are its heart and soul, and upon whom its success has been built. The impressive work of these community-based researchers demonstrates that with relatively modest investments in training, mentorship and pilot funding, investigators from LMIC can formulate innovative scientific questions of significant public health importance, develop rigorous, context-relevant research protocols and funding proposals, conduct the research necessary to study these questions, analyze and publish the results of their studies, and in the process advance our understanding of HIV prevention science for GMT populations in LMIC settings.

The pressing need for a training program that can raise the levels of HIV prevention knowledge and skills among GMT populations is grounded in the reality that many national public health programs in LMIC have consistently ignored or actively excluded MSM and transgender individuals from both HIV sentinel surveillance measures and coordinated local responses to HIV and AIDS. This reality, borne out of persistent, institutionalized homophobia and transphobia, has resulted in a longstanding and widespread lack of access to appropriate HIV prevention, treatment and care services for LGBT people in numerous countries. This in turn has produced a disproportionate and devastating burden of HIV morbidity and mortality among GMT populations in countless settings. Without focused efforts designed to address these disparities and increase the levels of local expertise available to prevent ongoing HIV transmission among GMT populations within these contexts, GMT people will continue to suffer HIV-related illness and death, efforts to end AIDS will fail in both individual countries and around the globe.

However, despite these appalling and avoidable challenges, it must be remembered that the history of the response to AIDS within gay communities nearly 
everywhere has been one of ongoing resilience and creativity that has contributed innovative weapons to combat the epidemic, and in the process pushed the field of HIV prevention science forward. This special issue demonstrates that the capacity to bravely and actively respond to immanent threat is also found among diverse GMT populations in countries throughout the world. The papers published in this special issue include data from research studies that were among the first to be attempted in their regions and sometimes the world:

- The paper by Gulov et al., [1] presents one of the first HIV seroprevalence surveys to be conducted among MSM in Central Asia and shows that community-based organizations are able to tap into sexual networks in ways that engage hidden HIV-positive men.

- The two papers by Hugo et al., [2, 3] present the first PEP uptake study to have been attempted on the African continent, along with data reporting awareness and uptake of PEP and PrEP among South Africa's remarkably diverse population of MSM.

- The papers by the CARMAH group in Vietnam [4-6] report findings from one of the first HIV and STI prevalence studies conducted among transgender women in Southeast Asia, and, consistent with the global literature, reveal sobering rates of HIV infection in this group and significant barriers to uptake of HIV testing and PrEP.

- The papers by Sapsirisavat et al., [7, 8] is the first from Southeast Asia to use CD4 assays as a means of describing the characteristics of late HIV testers who reside within the exploding HIV epidemic among MSM in Thailand, and to characterize barriers to testing in this population.

- Tohme et al., $[9,10]$ report data from Lebanon and is the first study in the world, to the best of our knowledge, on HIV prevalence and risk among MSM refugees from war zones.

Each of these papers demonstrates that there is an enormous pool of extraordinary talent and willingness to work hard to find ways to fight the epidemic within specific local contexts. The final papers-by Stahlman et al., [11] and by Stall et al., [12] respectively-provide a background for this group of studies within the larger literature on HIV health disparities among GMT populations in the developing world, and present a blueprint for a model program that can be used to develop capacity among GMT populations in LMIC settings, and so provide these populations with some of the tools they need to fight HIV/AIDS.

The unifying themes within the papers presented in this special issue can be summarized in a few sentences. GMT populations are highly over-represented in the HIV/AIDS caseload in countries around the globe and these HIV health disparities are often dramatic. In stark contrast, the HIV prevention and care needs of these individuals are routinely and willfully overlooked, allowing these epidemics to spread unchecked. It follows that we cannot end AIDS until we end HIV transmission among GMT populations around the world. While science-based HIV prevention and care efforts are necessary to address these global health disparities, they must be informed by, and respond to, specialized local contexts if they are to be effective tools in ending the epidemic. The insight needed to translate scientifically proven HIV prevention and care interventions so they can be effective at the local level requires the active participation of GMT populations and researchers. The essential contributions of communitybased participants to these efforts is enhanced by strategies to increase their scientific and public health knowledge and expertise so that GMT prevention workers can think globally but act locally.

The University of Pittsburgh/amfAR HIV Scholars program provides a model by which community-based workers committed to improving the health of GMT populations can be trained to conduct ground-breaking projects in their home countries, and, in the process build the skill sets that are required if we are to end the global HIV/AIDS epidemic among GMT populations. It is hoped that this and other programs like it will be sufficiently resourced to meet this growing challenge, and in the process, provide these populations with the tools that they need to end AIDS in their home countries, and ultimately, around the world.

\section{Compliance with Ethical Standards}

Conflict of Interest All authors have no conflicts of interest.

Ethical Approval This study did not involve human participants. Informed consent was not obtained.

\section{References}

1. Gulov K, Coulter RWS, Matthews DD, Uzzi M, Stall R. HIV and STIs among MSM in Tajikistan: laboratory-confirmed diagnoses and self-reported testing behaviors. AIDS Behav. 2016. doi:10. 1007/s10461-016-1457-z.

2. Hugo JM, Stall RD, Rebe K, Egan JE, Jobson G, De Swardt G, Struthers H, McIntyre JA. Knowledge, attitudes and beliefs regarding post exposure prophylaxis among South African men who have sex with men. AIDS Behav. 2016. doi:10.1007/s10461016-1520-9.

3. Hugo JM, Stall RD, Rebe K, Egan JE, De Swardt G, Struthers H, McIntyre JA. Anti-retroviral therapy based HIV prevention among a sample of men who have sex with men in Cape Town, South Africa: use of post-exposure prophylaxis and knowledge on pre-exposure prophylaxis. AIDS Behav. 2016. doi:10.1007/ s10461-016-1536-1.

4. Oldenburg CE, Le B, Toan T, Thien DD, Huyen HT, Friedman MR, Stall R, Colby D. HIV pre-exposure prophylaxis indication and readiness among HIV-uninfected transgender women in Ho 
Chi Minh City, Vietnam. AIDS Behav. 2016. doi:10.1007/ s10461-016-1483-x.

5. Bao A, Colby DJ, Trang T, Le BQ, Dinh TD, Nguyen QH, Hoang HT, Friedman MR, Stall R. Correlates of HIV Testing among transgender women in Ho Chi Minh, Vietnam. AIDS Behav. 2016. doi:10.1007/s10461-016-1574-8.

6. Colby D, Nguyen NA, Le B, Toan T, Thien DD, Huyen HT, Mai TDA, Friedman MR, Stall R. HIV and syphilis prevalence among transgender women in Ho Chi Minh City, Vietnam. AIDS Behav. 2016. doi:10.1007/s10461-016-1485-8.

7. Sapsirisavat V, Phanuphak N, Keadpudsa S, Egan JE, Pussadee K, Klaytong P, Friedman MR, van Griensven F, Stall R, The FAITH study team. Psychosocial and behavioral characteristics of high-risk men who have sex with men (MSM) of Unknown HIV Positive Serostatus in Bangkok, Thailand. AIDS Behav. 2016. doi:10.1007/s10461-016-1519-2.

8. Sapsirisavat V, Phanuphak N, Sophonphan J, Egan JE, Langevattana K, Avihingsanon A, Friedman MR, Stall R, The FAITH study team. Differences between men who have sex with men (MSM) with low CD4 cell counts at their first HIV Test and MSM with higher CD4 counts in Bangkok, Thailand. AIDS Behav. 2016. doi:10.1007/s10461-016-1456-0.
9. Tohme J, Egan JE, Stall R, Wagner G, Mokhbat J. HIV prevalence and demographic determinants of unprotected anal sex and HIV testing among male refugees who have sex with men in Beirut, Lebanon. AIDS Behav. 2016. doi:10.1007/s10461-0161484-9.

10. Tohme J, Egan JE, Friedman MR, Stall R. Psycho-social correlates of condom use and HIV testing among MSM refugees in Beirut, Lebanon. AIDS Behav. 2016. doi:10.1007/s10461-0161498-3.

11. Stahlman S, Beyrer C, Sullivan PS, Mayer KH, Baral SD. Engagement of gay men and other men who have sex with men (MSM) in the response to HIV: a Critical step in achieving an AIDS-Free generation. AIDS Behav. 2016. doi:10.1007/s10461016-1388-8.

12. Stall R, Egan JE, Kinsky S, Coulter RWS, Friedman MR, Matthews DD, Klindera K, Cowing M. Overview of the University of Pittsburgh/amfAR training program in HIV prevention research for MSM and male-to-female transgender populations in low and middle income countries. AIDS Behav. 2016. doi:10.1007/ s10461-016-1382-1. 